\title{
RENDIMENTO EM LAMINAÇÃO DE MADEIRA DE PARICÁ NA REGIÃO DE PARAGOMINAS, PARÁ
}

\author{
INCOME AND LOSS IN ROLLING OF PARICÁ WOOD IN THE REGION OF PARAGOMINAS, \\ PARÁ STATE
}

Gilson Fernandes da Silva ${ }^{1}$ Adriano Ribeiro de Mendonça ${ }^{2}$ Raphael Gomes Hoffmann ${ }^{3}$ Luciano Zumerli Zaneti ${ }^{4}$ José Franklim Chichorro ${ }^{5}$ Rinaldo Luiz Caraciolo Ferreira ${ }^{6}$

\section{RESUMO}

O objetivo deste estudo foi avaliar o rendimento em laminação de madeira de paricá (Schizolobium amazonicum Huber ex. Ducke) em plantios comerciais na região de Paragominas, Pará. A partir de 180 árvores-amostra oriundas de plantios com idades de cinco, seis e sete anos em espaçamento $4 \mathrm{x} 4 \mathrm{~m}$, foram avaliados o rendimento médio na atividade levando em consideração os rendimentos por idade e por classe de diâmetro. Foram avaliadas também as perdas em todo o processo de laminação. Os resultados apontaram um rendimento médio de 50,31\% na atividade, o que é compatível com espécies dos gêneros Pinus e Eucalyptus. Verificou-se que as árvores com maior idade e diâmetro apresentaram os maiores rendimento e, consequentemente, as menores perdas devido a defeitos e ao rolo-resto.

Palavras-chave: dendrometria; Schizolobium amazonicum; volume comercial.

\begin{abstract}
The aim of this study was to evaluate the performance of laminate wood paricá (Schizolobium amazonicum Huber ex. Ducke) in commercial plantations in the region of Paragominas, Pará state. Using 180 sample trees aged five, six and seven years,originating from plantations and grown in $4 \times 4 \mathrm{~m}$ spacing, the average income was evaluated, taking into account income by age and diameter class. Losses were also evaluated throughout the lamination process. The results show an average yield of 50.31\% in the activity, which is consistent with species of Pinus and Eucalyptus. Trees that were older and larger in diameter showed higher yield and therefore lower losses due to defects and the remainder roller.
\end{abstract}

Keywords: forest biometric; Schizolobium amazonicum; merchantable volume.

1 Engenheiro Florestal, Dr., Professor Associado III do Departamento de Ciências Florestais e da Madeira, Universidade Federal do Espírito Santo, Av. Governador Lindemberg, 316, Bairro Centro, CEP 29550-000, Jerônimo Monteiro (ES), Brasil. gilson.silva@pq.cnpq.br

2 Engenheiro Florestal, Dr., Professor Adjunto III do Departamento de Ciências Florestais e da Madeira, Universidade Federal do Espírito Santo, Av. Governador Lindemberg, 316, Bairro Centro, CEP 29550-000, Jerônimo Monteiro (ES), Brasil. adriano.mendonca@ufes.br

3 Engenheiro Florestal, M.Sc., Departamento de Ciências Florestais e da Madeira, Universidade Federal do Espírito Santo, Av. Governador Lindemberg, 316, Bairro Centro, CEP 29550-000, Jerônimo Monteiro (ES), Brasil. hoffmann84@hotmail.com

4 Engenheiro Agrônomo, MSc., Centro de Pesquisa do Paricá, BR 010, Km 16, CEP 68633-000, Dom Eliseu (PA), Brasil. lucianozaneti@yahoo.com.br

5 Engenheiro Florestal, Dr., Professor Associado III do Departamento de Ciências Florestais e da Madeira, Universidade Federal do Espírito Santo, Av. Governador Lindemberg, 316, Bairro Centro, CEP 29550-000, Jerônimo Monteiro (ES), Brasil. jfufes@gmail.com.br

6 Engenheiro Florestal, Dr., Professor Titular do Departamento de Ciência Florestal, Universidade Federal Rural de Pernambuco, Rua Manoel de Medeiros, s/n, Dois Irmãos, CEP 52171-900, Recife (PE), Brasil. rinaldo@dcfl.ufrpe.br

Recebido para publicação em 6/03/2012 e aceito em 4/09/2013 


\section{INTRODUÇÃO}

As lâminas de madeira podem ser definidas como um material produzido pela ação de corte, por meio de uma "faca específica", variando de 0,13 até $6,35 \mathrm{~mm}$ de espessura (IWAKIRI, 2005). O principal uso dessas é como matéria-prima para a indústria de compensado, cujos painéis são destinados à construção civil, fabricação de móveis, pisos e embalagens. As lâminas são utilizadas também na fabricação de fósforos e suas embalagens, palitos para sorvetes, revestimentos de móveis e outras superfícies (TSOUMIS, 1991).

No Brasil, as primeiras laminadoras e fábricas de compensados foram instaladas na década de 30. Inicialmente, a matéria-prima utilizada era originária de espécies nativas da floresta amazônica e de espécies da floresta atlântica, onde havia exploração da madeira sem a devida reposição da mesma. Contudo, com o passar das décadas, houve a necessidade de utilização de matéria-prima originária de florestas plantadas devido à legislação mais rigorosa em relação ao uso das florestas nativas.

No Sul do país, a madeira de pinus começou a ser utilizada em escala comercial, tornando-se a principal matéria-prima para produção de lâminas e compensados. Na região Sudeste, o eucalipto tem se tornado uma espécie promissora na produção de lâminas de madeira por meio do melhoramento genético. Já na região Norte, o paricá (Schizolobium amazonicum Huber ex. Ducke), uma espécie nativa da região amazônica, passou a ser muito utilizada para produção de lâminas e compensados. Com isso, vários pesquisadores têm feito esforços para gerar mais informações com o intuito de melhorar o conhecimento sobre a silvicultura e o manejo dos plantios (PERES FILHO et al., 2002, RONDON, 2002; CARVALHO, 2005; ROSA et al., 2009; OHASHI et al., 2010; ALVINO-RAYOL et al., 2011) e as características tecnológicas e utilização da madeira (IWAKIRI et al., 2010a; IWAKIRI et al., 2010b; TEREZO e SZÜCS, 2010; ARRUDA et al., 2011) desta espécie.

Um dos problemas encontrado pelas empresas do setor de laminação está no aproveitamento da madeira (rendimento). De acordo com Gerwing et al. (2001), fatores como estocagem inadequada, secagem não uniforme e equipamentos inadequados podem contribuir significativamente para a redução do rendimento. Mendes et al. (2000) afirma que o baixo nível tecnológico dos equipamentos empregados, associado com a elevada idade média destes e a carência de técnicas modernas e especializadas, contribui para um baixo rendimento. Além disso, fatores como características do fuste (diâmetro e forma), tensões de crescimento, direção da grã (ALMEIDA, 2011), velocidade de corte, ajuste da faca e barra de pressão, afiação da faca, processo de aquecimento das toras (LUTZ, 1978 citado por ALMEIDA, 2011) podem afetar o rendimento em laminação.

Assim, para um aumento na produtividade, é essencial a busca por madeiras que tenham maior fator de forma, maior diâmetro da tora e menor rolo-resto. Bonduelle et al. (2006) verificaram que a conicidade foi a característica que teve maior correlação com o rendimento de laminação de Pinus spp. comparado aos diâmetros das extremidades e diâmetro médio da tora, volume laminado e volume total. Estes autores também citam que o tamanho do torno não teve influência no rendimento das toras em lâminas verdes. Já Mendes et al. (2000) destacaram os cuidados no manuseio e preparação das toras no que se refere a condições de armazenamento, conversão das toras e acondicionamento das mesmas para laminação, além de critérios adequados quanto à seleção, preparação/ajuste, operação e manutenção dos equipamentos. Gerwing et al. (2001), avaliando rendimento de madeira no estado do Pará, cita que $\mathrm{o}$ armazenamento inadequado das toras resultou em danos causados por insetos e rachadura da tora, com perdas de $8 \%$ e $13 \%$, respectivamente.

Considerando o exposto, um maior aproveitamento desde o corte da madeira até a obtenção das lâminas é desejado. Assim, este presente trabalho teve como objetivo avaliar a influência da idade e diâmetro das árvores no rendimento em laminação da madeira do paricá, bem como as perdas ocorrentes no processo de laminação.

\section{MATERIAIS E MÉTODOS}

\section{Descrição da área de estudo}

Os dados para este estudo foram coletados em povoamentos homogêneos de Schizolobium amazonicum Huber ex. Ducke, situados entre os municípios de Dom Eliseu e Paragominas, Estado do Pará, pertencentes a empresas ligadas ao Centro de Pesquisa do Paricá (CPP), com idades de cinco, seis e sete anos e com espaçamento de $4 \times 4$ metros. 


\section{Medição do volume laminado e do rendimento da laminação}

Para a realização do trabalho foram escolhidas duas áreas de plantio conduzidas sob as mesmas condições de manejo (mesmo sítio, espaçamento 4 x $4 \mathrm{~m}$, mesmas condições de plantio e fertilização), mas em três idades diferentes, isto é, 5, 6 e 7 anos de idade. Em cada um dessas áreas e em cada idade, foi selecionada ao acaso uma parcela de $512 \mathrm{~m}^{2}$ e todas as árvores vivas da parcela foram abatidas para a coleta dos dados. Assim, foram consideradas duas parcelas em cada idade, totalizando seis parcelas. Em função disso e considerando a mortalidade ocorrida em cada uma das parcelas, foram utilizadas 180 árvores $(62,57 \mathrm{e}$ 61 árvores-amostra, respectivamente, para as idades de cinco, seis e sete anos), conforme detalhado na Tabela 1.

As árvores foram derrubadas, retirando-se a copa e as ramificações existentes e medindo-se o fuste até um diâmetro mínimo de $10 \mathrm{~cm}$. Após esta etapa, cada tora foi dividida em toretes de $1,38 \mathrm{~m}$ de comprimento, dimensão normalmente utilizada pela empresa, uma vez que o torno empregado para a produção de lâminas apresenta $1,5 \mathrm{~m}$ de boca.

Os toretes produzidos para cada árvore foram devidamente identificados, o que permitiu rastrear a que árvore os toretes pertenciam e como foi o seu rendimento na produção de lâminas na sequência do processo. Em cada torete, mediramse os diâmetros no centro e nas extremidades, procedendo-se ao cálculo do volume cubado pelo método de Newton. Assim, o volume deixado no campo para cada árvore-amostra foi obtido pela diferença entre o volume total do fuste até um diâmetro mínimo de $10 \mathrm{~cm}$ e o somatório dos volumes dos toretes de $1,38 \mathrm{~m}$ de comprimento de cada árvore (1).

$$
V \text { campo }_{j}=V T_{j}-V T T_{j}
$$

Em que: Vcampo $_{j}=$ volume deixado no campo para a j-ésima árvore-amostra após o corte dos toretes, $\mathrm{em} \mathrm{m}^{3} ; V T_{j}=$ volume total do fuste cubado no campo para a j-ésima árvore-amostra até o diâmetro mínimo de $10 \mathrm{~cm}, \mathrm{em} \mathrm{m}^{3} ; V T T_{j}=$ Volume total dos toretes cubados para a j-ésima árvore-amostra, isto é, $V T T_{j}=\sum_{i=1}^{n} V_{\text {Torete }_{i}}$, em que VTorete ${ }_{\mathrm{i}}$ corresponde ao volume do i-ésimo torete, relativo à j-ésima árvore-amostra, $\mathrm{em}^{3}$.

Aproveitando-se das definições de cálculo ora apresentadas, definiu-se o volume total do fuste cubado no campo até um diâmetro mínimo de 10 $\mathrm{cm}(V T)$ para todas as árvores em uma mesma idade. Esta informação foi útilizada para o cálculo do volume relativo de perdas e o rendimento da laminação, e foi obtida pela seguinte expressão:

$$
V T=\sum_{j=1}^{n} V T_{j}
$$

Em que $n=62,57,61$, respectivamente, para as idades de 5, 6 e 7 anos.

Após esta etapa, os toretes foram carregados e transportados até a fábrica, onde passaram pela fase de laminação. Nesta fase, as lâminas na saída do torno tinham diferentes tamanhos, sendo obtidos vários comprimentos, variando em 2,$76 ; 1,45 ; 0,98 ; 0,66 ; 0,45 ; 0,38$ e 0,29 $\mathrm{m}$, com largura de $1,38 \mathrm{~m}$ e espessura de $2,2 \mathrm{~mm}$. Dessa forma, foi possível obter o volume de cada lâmina separadamente pela fórmula apresentada por Mendes et al., (2000) (3).

TABELA 1: Distribuição de frequência das árvores por idade e classes de diâmetro em plantios de paricá situados na região de Paragominas - PA.

TABLE 1: Frequency distribution of trees by age and diameter classes in paricá plantation in the region of Paragominas, Pará state, Brazil.

\begin{tabular}{cccccccccccc}
\hline \multirow{2}{*}{ Idade (anos) } & \multicolumn{10}{c}{ Classes de diâmetro $(\mathrm{cm})$} & \multirow{2}{*}{ Total } \\
\cline { 2 - 10 } & 11 & 13 & 15 & 17 & 19 & 21 & 23 & 25 & 27 & 29 & \\
\hline 5 & - & 5 & 8 & 9 & 15 & 17 & 7 & 1 & - & - & 62 \\
6 & - & 5 & 5 & 8 & 11 & 10 & 8 & 6 & 2 & 2 & 57 \\
7 & 1 & 5 & 5 & 5 & 8 & 10 & 11 & 9 & 4 & 3 & 61 \\
\hline Total & 1 & 15 & 18 & 22 & 34 & 37 & 26 & 16 & 6 & 5 & 180 \\
\hline
\end{tabular}




$$
\operatorname{VLam}_{i}=L^{*} C_{i} * e
$$

Em que: $V \operatorname{Lam}_{i}=$ volume da i-ésima lâmina, em m ${ }^{3} ; L=$ largura das lâminas; $C_{i}=$ comprimento da i-ésima lâmina, em m; em m; $e=$ espessura das lâminas, em m.

Admitindo que todos os toretes de uma mesma árvore-amostra foram laminados e o volume de cada lâmina calculado a partir da expressão (3), o volume total laminado para cada árvore-amostra foi obtido, então, pelo somatório do volume das lâminas (4).

$$
\operatorname{VTLam}_{j}=\sum_{i=1}^{n} V \operatorname{Lam}_{i}
$$

Em que: $\operatorname{VTLam}_{j}=$ volume total laminado para a j-ésima árvore-amostra, considerando todos os toretes trazidos do campo, em $\mathrm{m}^{3}$.

Para auxiliar a obtenção do rendimento da laminação, o cálculo do volume total laminado (VTLam) para todas as árvores em uma mesma idade, foram obtidas por meio da expressão (5):

$$
\text { VTLam }=\sum_{j=1}^{n} V_{\text {Lam }}
$$

Em que $n=62,57,61$, respectivamente, para as idades de 5, 6 e 7 anos.

O cálculo do rendimento para cada idade foi obtido pela expressão (6).

$$
\text { Rend }=\frac{\text { VTLam }}{V T} 100
$$

\section{Avaliação do rendimento médio em laminação por idade e classe diamétrica}

O rendimento foi considerado como a percentagem de aproveitamento de madeira laminada que foi gerada no processo desde a derrubada das árvores até a obtenção do produto final, que foram as lâminas. Assim, com o objetivo de se avaliar o efeito da idade no rendimento em laminação, foi realizado o cálculo do rendimento para todas as árvores nas três idades, a fim de se verificar o aproveitamento de madeira na atividade. $\mathrm{O}$ cálculo do rendimento de cada árvore em cada idade foi feito dividindo-se o volume total laminado da árvore $\left(\right.$ VTLam $\left._{j}\right)$ pelo volume total do fuste até o diâmetro mínimo de $10 \mathrm{~cm}$ da respectiva árvore $\left(V T_{j}\right)$, multiplicando-se o resultado desta razão por 100 .

Para avaliar se as médias dos rendimentos diferiram estatisticamente entre as idades, foi realizado o teste $t$ de Student comparando-se as idades. Para efeito dessa comparação, as três idades foram dividas em pares, obtendo-se um total de três combinações ( 5 e $6 ; 6$ e 7 e 5 e 7 anos). Para a correta aplicação do teste $t$ de Student, foi realizado um teste $F$ preliminar para verificar se as variâncias eram homogêneas ou não, determinando assim o teste adequado para cada caso. As análises foram realizadas considerando para todos os cálculos um nível de $5 \%$ de significância $(p \leq 0,05)$.

Foi realizada também uma análise de regressão para verificar a influência das classes de diâmetro no rendimento em laminação nas três idades. Para tal, foram testados sete modelos estatísticos: linear, quadrático, cúbico, raiz quadrada, hiperbólico, logarítmico e cúbico raiz ajustados, considerando para a avaliação da significância dos parâmetros um nível de $5 \%$ de probabilidade $(p$-valor $\leq 0,05)$. Os modelos foram avaliados por meio do erro padrão da estimativa $\left(S_{y, x}\right)$, erro padrão relativo $\left[S_{y, x}(\%)\right]$ e coeficiente de determinação ajustado ( $\bar{R}^{2}$ Ajust.).

\section{Medição das perdas no processo de laminação}

Assim como ocorreram perdas de volume no campo com a divisão do fuste de cada árvore em toretes, também ocorreram perdas com o processo de laminação. Estas perdas acontecem basicamente a partir de duas causas principais: perdas devido à presença do rolo-resto; e defeitos na madeira devido à maior ou menor presença de fendas e nós e, também, devido à tortuosidade natural apresentada pelos toretes.

$\mathrm{O}$ volume devido à presença do rolo-resto foi calculado a partir da expressão (7).

$$
V R R_{i}=\frac{\pi}{40000} d^{2} L
$$

Em que: $V R R_{i}=$ volume do i-ésimo rolo-resto, em $\mathrm{m}^{3} ; d \stackrel{i}{=}$ diâmetro na metade do comprimento do rolo-resto, em $\mathrm{cm} ; L=$ comprimento do rolo-resto, em $\mathrm{m}$.

O volume total de rolo-resto para cada árvore-amostra foi calculado tal como se segue: 


$$
V T R R_{j}=\sum_{i=1}^{n} V R R_{i}
$$

Em que: $V T R R_{j}=$ volume total de rolo-resto para a j-ésima árvore-amostra, considerando todos os toretes trazidos do campo, em $\mathrm{m}^{3}$.

Com isso, foi possível calcular o total de perdas e os três tipos de perdas ocorridos no processo de laminação, em termos absolutos e relativos. Estes cálculos foram feitos para cada uma das idades. As perdas relativas foram calculadas em função do volume total de perdas ( $T P$, variável definida a seguir) e do volume total do fuste até um diâmetro mínimo de $10 \mathrm{~cm}(V T)$. Assim, os cálculos foram realizados de acordo com o que se segue:

a) Volume total de perdas no processo de laminação $(T P), \mathrm{em} \mathrm{m}^{3}$ :

$$
T P=\sum_{j=1}^{n}\left(V T_{j}-V T L a m_{j}\right)
$$

Em que $n=62,57,61$, respectivamente, para as idades de 5, 6 e 7 anos.

b) Volume total de perdas no campo (PC), em $\mathrm{m}^{3} \mathrm{e}(\%)$ :

$$
P C=\sum_{j=1}^{n} \text { Vcampo }_{j}
$$

$$
P C(\%)=\frac{P C}{T P} 100 \quad \text { ou } \quad P C(\%)=\frac{P C}{V T} 100
$$

c) Perdas na laminação devido ao rolo-resto $(P R R), \mathrm{em} \mathrm{m}^{3} \mathrm{e}(\%)$ :

$$
P R R=\sum_{j=1}^{n} V V T R R_{j}
$$

$P R R(\%)=\frac{P R R}{T P} 100$ ou $P R R(\%)=\frac{P R R}{V T} 100$

d) Perdas na laminação devido a defeitos na madeira $(P D), \mathrm{em} \mathrm{m}^{3} \mathrm{e}(\%)$ :

$$
\begin{gathered}
P D=\sum_{j=1}^{n}\left(V T_{j}-\text { Campo }_{j}-V T L a m_{j}-V T R R_{j}\right) \\
P D(\%)=\frac{P D}{T P} 100 \text { ou } P D(\%)=\frac{P D}{V T} 100
\end{gathered}
$$

\section{RESULTADOS E DISCUSSÃO}

\section{Avaliação do rendimento em laminação}

Pela observação da Tabela 2, é possível verificar que, na idade de sete anos, obteve-se o melhor rendimento em laminação. Isso pode ser explicado pelo fato das árvores nesta idade apresentarem maior fator de forma, conforme encontrado em Hoffmann et al. (2011), e maiores diâmetros. O rendimento, considerando-se as árvores das três idades, foi de $50,31 \%$, próximo a valores encontrados na literatura para espécies dos gêneros Pinus e Eucalyptus, conforme observado por Almeida et al. (2004) e Bonduelle et al. (2006).

Brand et al. (2004), estudando o comportamento do rendimento de espécies do gênero Pinus em laminação de toras sem casca em lâminas verdes, obtiveram rendimento médio de 40,71\% (volume de lâmina seca classificada que sai da laminadora/volume de toras). Bonduelle et al. (2006) encontraram resultados de rendimento médio em laminação de Pinus spp, com casca, em torno de 48\%. Bortoleto Júnior (2008) encontrou um rendimento médio de $54,4 \%$ para produçao de lâminas de árvores com casca de Pinus merkusii. Coneglian et al. (2009), estudando o efeito do tratamento térmico com vapor no processo de laminação, encontraram o rendimento médio de 34,55\% em Eucalyptus grandis. Almeida (2011) encontrou rendimento de toras sem casca de $52,74 \%$ e com casca de $28,96 \%$, para laminação do híbrido de Pinus elliotti var. elliotti x Pinus caribaea var. hondurensis

Para o paricá, ficou evidenciado que esta espécie pode propiciar rendimentos em laminação semelhantes ou até superiores às espécies dos gêneros Pinus e Eucalyptus mostrando-se como uma espécie competitiva neste mercado face seu rápido crescimento e qualidade da madeira para o uso em laminação.

\section{Avaliação do rendimento médio em laminação por idade e classe diamétrica}

Analisando os resultados apresentados na Tabela 2, verifica-se que o teste $t$ foi significativo apenas para o par 5 e 7 anos, o que evidencia que a idade foi uma variável influente no rendimento da laminação. Assim, a busca pela idade ótima para corte de povoamentos manejados para produção de madeira para laminação é um aspecto relevante na 
TABELA 2: Rendimento por idade após o processo de laminação da madeira de paricá em plantios situados na região de Paragominas - PA, em que $V T L a m=$ volume total laminado e $V T=$ volume total.

TABLE 2: Income and losses by age after the lamination process of wood from paricá plantations in the region of Paragominas, PA state, where VTLam = laminate total volume and $V T=$ total volume.

\begin{tabular}{cccc}
\hline Idade (anos) & $V T L a m\left(\mathrm{~m}^{3}\right)$ & $V T\left(\mathrm{~m}^{3}\right)$ & Rendimento (\%) \\
\hline 5 & 8,01 & 18,29 & $43,79 \mathrm{a}$ \\
6 & 9,71 & 18,89 & $51,40 \mathrm{ab}$ \\
7 & 15,11 & 27,11 & $55,74 \mathrm{~b}$ \\
\hline
\end{tabular}

tomada de decisão.

Por outro lado, admitindo que em idades maiores haja uma chance maior de se encontrar árvores com maiores diâmetros, é razoável pensar então que, na verdade, o diâmetro pode ser o agente direto que provocou o aumento de rendimento. Para checar esta hipótese, foram ajustadas regressões relacionando o rendimento em laminação da madeira do paricá com o diâmetro das árvores laminadas. Após ajustar os modelos linear, quadrático, cúbico, raiz quadrada, hiperbólico, logarítmico e cúbico raiz e considerando as medidas de precisão erro padrão da estimativa, absoluto e relativo $\left(S_{y, x}\right.$ e $S_{y, x}$ (\%), respectivamente), coeficiente de determinação ajustado ( $\bar{R}^{2}$ Ajust.) e a avaliação da significância dos parâmetros a um nível de $5 \%$ de probabilidade $(p$-valor $\leq 0,05)$ pelo teste $t$, concluiu-se que o modelo linear foi o que melhor representou a tendência de variação do rendimento em função do diâmetro.

A Figura 1 se observa que a variação média do rendimento em laminação em função da variação no tamanho das classes de diâmetro e por meio desta figura pode-se verificar que o modelo linear realmente teve um desempenho muito satisfatório para representar as variações mencionadas. Os demais modelos avaliados apresentaram contribuições insignificantes em termos de precisão em relação ao modelo linear, e por ser este um modelo mais simples, optar por este modelo tornou-se uma escolha relativamente óbvia. Assim, pode-se afirmar que o modelo linear parece ser uma boa escolha para expressar a relação entre o rendimento da laminação e o diâmetro das árvores e, admitindo este fato, nota-se que, para cada acréscimo unitário em DAP, ocorre um acréscimo médio estimado de aproximadamente $2,6 \%$ no rendimento em laminação. Com isso, podese concluir que o diâmetro tem influência sobre o rendimento na laminação da madeira de paricá.
Uma explicação para este resultado está no fato de que o volume de rolo-resto é aproximadamente constante, isto é, à medida que a árvore cresce em volume total, o volume do rolo-resto permanece aproximadamente o mesmo. Com isso, as perdas devido ao rolo-resto tendem a se diluir em árvores de maior diâmetro (maior diâmetro está altamente correlacionado o maior volume), o que aumenta o rendimento em laminação.

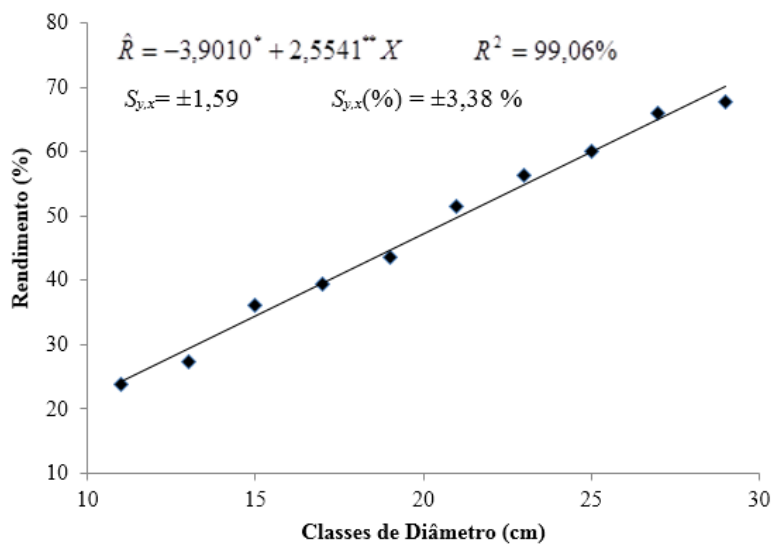

FIGURA 1: Variações do rendimento em laminação da madeira do paricá $(\hat{R})$ em função do tamanho da classe de diâmetro $(X)$, em que ${ }^{*} \mathrm{e}^{* *}$ correspondem a significativo, em nível de $1 \%$ e $5 \%$ de probabilidade, respectivamente, pelo teste $t$.

FIGURE 1: Variations in lamination yield of paricá wood $(\hat{R})$ as a function of the class size diameter $(X)$ wherein $*$ and $* *$ represent significant level of $1 \%$ to $5 \%$ probability, respectively, by t-test.

\section{Perdas no processo de laminação}

De acordo com os resultados apresentados na Tabela 3, quando se analisam as perdas relativas 
em relação ao volume total de perdas $(T P)$, pode-se observar que na idade de sete anos se encontraram menores perdas relativas por defeitos e devido ao rolo-resto. A maior fonte de perda foi o volume deixado no campo. Isto ocorreu especialmente para o caso do rolo-resto, uma vez que na idade de sete anos existem árvores maiores, e há uma tendência do volume de rolo-resto se diluir com o aumento do tamanho das árvores, como anteriormente mencionado. Bonduelle et al. (2006) citam que quanto menor o diâmetro da tora, menor será a faixa de aproveitamento em laminação, até atingir o diâmetro do rolo-resto.

Quando se analisam as perdas relativas em relação ao volume total $(V T)$, nota-se uma tendência de redução das perdas no rolo-resto e por defeitos com o aumento da idade, o que não acontece com as perdas no campo. Por outro lado, as perdas no campo vão depender da combinação do comprimento do fuste até $10 \mathrm{~cm}$ de diâmetro e de quantos toretes de 1,38 metros se podem retirar deste fuste. A título de exemplo, para uma árvore com 10 metros de comprimento do fuste, considerando-se o diâmetro da ponta mais fina igual a $10 \mathrm{~cm}$, pode-se retirar 7 (sete) toras de $1,38 \mathrm{~m}$. Com isso, seriam aproveitados 9,66 m, ocasionando uma perda de $0,34 \mathrm{~m}$ do comprimento útil. Algumas pesquisas estudaram a otimização do traçamento das árvores. Arce et al. (2004) avaliaram uma metodologia de otimização de corte, ou traçamento de árvores e chegaram à redução de $16 \%$ para $5 \%$ dos resíduos deixados na floresta. Mas, no trabalho citado, foram considerados comprimentos de toras diferentes, assim como no trabalho de Mendonça et al. (2008), diferentemente da presente pesquisa, em que o comprimento é padronizado $(1,38 \mathrm{~m})$, tornando este tipo de perda mais difícil de controlar.

Em face desses resultados e pensando no manejo da floresta no sentido de maximizar o rendimento em laminação, estratégias deveriam ser adotadas buscando-se reduzir as perdas ora avaliadas. Quanto às perdas relativas no campo, não há muito que possa ser feito. Perdas relativas por rolo-resto podem ser minimizadas por meio de práticas de silvicultura e manejo, como a escolha de espaçamentos mais adequados, realização de desbastes e realização de inventários contínuos no sentido de monitorar o crescimento em diâmetro para se escolher idades mais adequadas de corte. Por outro lado, as perdas relativas que parecem mais sensíveis à redução a partir de práticas de manejo são aquelas devidas a defeitos. Estas perdas parecem sofrer influência da idade de corte, o que pode ser uma decisão do manejador florestal. O aumento da rotação também pode colaborar na redução da presença de fendas, conferindo à madeira características tecnológicas mais desejáveis. Isto ocorre porque em árvores com rotações mais curtas existe uma maior quantidade de madeira juvenil e também uma tendência a tensões de crescimento mais elevadas. A melhor forma da árvore (menor conicidade e ausência de tortuosidade) também deve ser considerada nas práticas de manejo, o que pode ser obtido com melhoramento genético e com a escolha de espaçamentos mais adequados. Todas essas possibilidades merecem maior esforço de pesquisa para se aumentar o rendimento em laminação do paricá.

Confrontando os resultados aqui encontrados com os de outros trabalhos análogos, no estudo de produção de lâminas em dois clones de Eucalyptus, Almeida et al. (2004) encontraram

TABELA 3: Perdas após o processo de laminação da madeira de paricá em plantios com diferentes idades situados na região de Paragominas - PA.

TABLE 3: Losses after the lamination process of wood of paricá in plantations with different ages in the region of Paragominas, PA state.

\begin{tabular}{|c|c|c|c|c|c|c|c|c|c|c|}
\hline \multirow[t]{2}{*}{ Idade (anos) } & \multicolumn{4}{|c|}{ Perdas Absolutas $\left(\mathrm{m}^{3}\right)$} & \multicolumn{3}{|c|}{$\begin{array}{c}\text { Perdas Relativas a } \\
\qquad V T(\%)\end{array}$} & \multicolumn{3}{|c|}{$\begin{array}{c}\text { Perdas Relativas a } \\
\text { TP }(\%)\end{array}$} \\
\hline & $P C$ & $P D$ & PRR & $T P$ & $P C$ & $P D$ & $P R R$ & $P C$ & $P D$ & $P R R$ \\
\hline 5 & 3,11 & 5,60 & 1,57 & 10,28 & 17,00 & 30,62 & 8,58 & 30,25 & 54,47 & 15,27 \\
\hline 6 & 2,09 & 5,44 & 1,65 & 9,18 & 11,06 & 28,80 & 8,73 & 22,77 & 59,26 & 17,97 \\
\hline 7 & 6,13 & 4,14 & 1,73 & 12,00 & 22,61 & 15,27 & 6,38 & 51,08 & 34,50 & 14,42 \\
\hline
\end{tabular}

Em que: $\mathrm{VT}=$ volume total; $\mathrm{TP}=$ volume total de perdas no processo de laminação; $\mathrm{PC}=$ volume total de perdas no campo; PD = Pernas na laminação devido a defeitos na madeira; PRR = perdas na laminação devido ao rolo-resto. 
perdas médias de rendimento devido ao rolo-resto na ordem de $13,81 \%$ e $10,92 \%$ para cada clone, aos nove anos de idade. Estes mesmos autores verificaram, em outras literaturas, que as perdas médias de rendimento em rolo-resto para outras espécies do gênero Eucalyptus foi da ordem de $21,42 \%$, variando de 12,77 e $32,93 \%$, indicando a potencialidade do paricá para produção de lâminas.

\section{CONCLUSÕES}

Considerando os resultados encontrados, pode-se concluir que:

O paricá apresentou rendimento médio em laminação da madeira semelhante às espécies tradicionais como pinus e eucalipto, indicando ser uma espécie com potencial para esse fim.

A idade demonstrou exercer influência direta no rendimento em laminação, quer seja por apresentar árvores com maior diâmetro, quer seja por em idades mais avançadas haver uma tendência de menor ocorrência de defeitos pelo fato da madeira ter uma tendência em apresentar melhores características tecnológicas.

A variável diâmetro exerce influência direta no rendimento em laminação e o rendimento em laminação do paricá pode ser estimado com eficácia utilizando os diâmetros das árvores, havendo um acréscimo médio de rendimento aproxidamente constante em função de acréscimos unitários do diâmetro.

\section{AGRADECIMENTOS}

À Fundação de Apoio à Ciência e Tecnologia do Espírito Santo (FAPES), pela concessão da bolsa de estudos.

\section{REFERÊNCIAS BIBLIOGRÁFICAS}

ALMEIDA, R. R.; BORTOLETTO JÚNIOR, G.; JANKOWSKY, I. P. Produção de lâminas a partir da madeira de clones do híbrido Eucalyptus grandis $\mathrm{x}$ Eucalyptus urophylla. Scientia Forestalis, Piracicaba, n. 65, p. 49-58. 2004.

ALMEIDA, N. F. de. Avaliação da qualidade da madeira de um híbrido de Pinuselliotti var. elliotti $\mathrm{x}$ Pinuscaribaea var. hondurensis para a produção de lâminas e manufatura de compensados. 2011. 115 p. Dissertação (Mestrado em Recursos Florestais) - Universidade de São Paulo, Piracicaba, 2011.
ALVINO-RAYOL, F. de O.; ROSA, L. dos S.; RAYOL, B. P. Efeito do espaçamento e do uso de leguminosas de cobertura no manejo de plantas invasoras em reflorestamento de Schizolobium amazonicum Huber ex Ducke (paricá). Revista Árvore, v. 35, n. 3, p. 391-399, 2011.

ARCE, J. E.; MACDONAGH, P.; FRIEDL. R. A. Geração de padrões ótimos de corte através de algoritmos de traçamento aplicados a fustes individuais. Revista Árvore, v. 28, n. 2, p. 207-217. 2004.

ARRUDA, L. M. et al. Estudo preliminar das propriedades de compensados produzidos com lâminas de paricá (Schizolobium amazonicum Huber ex Ducke) modificadas termomecanicamente. Ciência da Madeira, v. 2, n. 1, p. 29-42. 2011.

BONDUELLE, G. M. et al. Estudo sobre os fatores que influenciam no rendimento em laminação de Pinus sp. Floresta e Ambiente, v. 12, n. 2, p. 35-41. 2006.

BORTOLETO JÚNIOR, G. Avaliação da qualidade da madeira de Pinus merkusii. Scientia Forestalis. v. 36, n. 78, p. 95-103. 2008.

BRAND, M. A. et al. Avaliação do processo produtivo de uma indústria de manufatura de painéis por meio do balanço de material e do rendimento da matéria-prima. Revista Árvore. v. 28, n. 4, p. 553-562. 2004.

CARVALHO, C. J. R. de. Respostas de plantas de Schizolobium amazonicum [S. parahyba var. amazonicum] e Schizolobium parahyba [Schizolobium parahybum] à deficiência hídrica. Revista Árvore, v. 29, n. 6, p. 907-914. 2005.

CONEGLIAN, A; SEVERO, E. T. D.; BORTOLETTO JÚNIOR, G. Avaliação do processo de laminação de toras de Eucalyptus grandis, utilizando o tratamento térmico com vapor. Energia na Agricultura. v. 24, n. 2, p. 1-13. 2009.

GERWING, J. et al. Rendimento no processamento de madeira no Estado do Pará. Belém: IMAZON, 38 p. 2001. (Série Amazônica n. 18).

HOFFMANN, R. G. et al. 2011. Caracterização dendrométrica de plantios de paricá (Schizolobium amazonicum Huber ex. Ducke) na região de Paragominas, PA. Revista Brasileira de Ciências Agrárias, v. 6, n. 4, p. 675-684. 2011.

IWAKIRI, S. Painéis de madeira reconstituída. Curitiba: FUPEF, 2005.

IWAKIRI, S. et al. Produção de painéis laminados unidirecionais - LVL com lâminas de Schizolobium amazonicum, Eucalyptus saligna e Pinus taeda. 
Cerne. v. 16, n. 4, p. 557-563. 2010a.

IWAKIRI, S. et al. Avaliação do potencial de utilização da madeira de Schizolobium amazonicum "Paricá" e Cecropia hololeuca "Embaúba" para produção de painéis aglomerados. Acta Amazônica. v. 40, n. 2, p. 303-308. 2010 b.

MENDES, M. M.; ALBUQUERQUE, C. E. C. de; IWAKIRI, S. Procedimento prático para cálculo de produção de lâminas de madeira por desenrolamento. Lavras: UFLA, 2000. 21 p. (Boletim Agropecuário).

MENDONÇA, A. R. de et al. Avaliação de um sistema para otimização do sortimento de Eucalyptus sp. Ciência Florestal, v. 18, n. 2, p. 247258. 2008.

OHASHI, S. T.; YARED, J. A. G.; FARIAS NETO, J. T. de. Variabilidade entre procedências de paricá Schizolobium parahyba var amazonicum (Huber ex Ducke) Barneby plantadas no município de Colares - Pará. Acta Amazônica. v. 40, n. 1, p. 8188. 2010.

PERES FILHO, O.; DORVAL, A.; BERTI FILHO, E. Preferência de saúva limão, Atta sedens riubropilosa Forel, 1908 (Hymenoptera, Formicidae) a diferentes espécies florestais, em condições de laboratório. Ciência Florestal. v. 12, n. 2, p. 1-7. 2002.

RONDON, E. V. Produção de biomassa e crescimento de árvores de Schizolobium amazonicum (Huber) Ducke sob diferentes espaçamentos na região de mata. Revista Árvore. v. 26, n. 5,p. 573-576. 2002.

ROSA, L. dos S. et al. Emergência, crescimento e padrão de qualidade de mudas de Schizolobium amazonicum Huber ex. Ducke sob diferentes níveis de sombreamento e profundidades de semeadura. Revista Ciências Agrárias. n. 52, p. 87-98. 2009.

TEREZO, R. F.; SZÜCS, C. A. Análise de desempenho de vigas em madeira laminada colada de paricá (Schizolobium amazonicum Huber ex. Ducke). Scientia Forestalis. n. 87, p. 471-480. 2010.

TSOUMIS, G. Science and technology of wood: structure, properties, utilization. Van Nostrand Reinhold, 1991. 494 p. 